\title{
Regularity of Global Attractors for the Kirchhoff Wave Equation
}

\author{
Xiaoyi Liu, Ping Gao \\ College of Mathematics and Information Science, Guangzhou University, Guangzhou, China \\ Email: 443205132@qq.com
}

How to cite this paper: Liu, X.Y. and Gao, P. (2019) Regularity of Global Attractors for the Kirchhoff Wave Equation. Journal of Applied Mathematics and Physics, 7, 2481-2491.

https://doi.org/10.4236/jamp.2019.710168

Received: September 29, 2019

Accepted: October 21, 2019

Published: October 24, 2019

Copyright (c) 2019 by author(s) and Scientific Research Publishing Inc. This work is licensed under the Creative Commons Attribution International License (CC BY 4.0).

http://creativecommons.org/licenses/by/4.0/

\begin{abstract}
In this paper, we mainly use operator decomposition technique to prove the global attractors which in $H_{0}^{1} \times L^{2}$ for the Kirchhoff wave equation with strong damping and critical nonlinearities, are also bounded in $H^{2} \times H_{0}^{1}$.
\end{abstract}

\section{Keywords}

The Kirchhoff Wave Equation, Critical Exponent, The Regularity of Global Attractor

\section{Introduction}

In this paper, we discuss the regularity of global attractors for the following Kirchhoff wave equation

$$
\begin{gathered}
u_{t t}-\left(1+\epsilon\|\nabla u\|^{2}\right) \Delta u-\Delta u_{t}+f\left(u_{t}\right)+h(u)=g(x) \quad \text { in } \Omega \times \mathbb{R}^{+}, \\
\left.u\right|_{\partial \Omega}=0, \quad u(x, 0)=u_{0}(x), \quad u_{t}(x, 0)=u_{1}(x), \quad x \in \Omega,
\end{gathered}
$$

where $\Omega$ is a bounded domain in $\mathbb{R}^{3}$ with the smooth boundary $\partial \Omega$, $\epsilon \in[0,1], f(m)$ and $h(m)$ are nonlinear functions and $g(x)$ is an external force term which is independent of time.

G. Kirchhoff [1] introduced the Equation (1.1) in $\mathbb{R}^{1}$ without dissipation $-\Delta u_{t}$ and nonlinear perturbations $f\left(u_{t}\right)$ and $h(u)$, and described the oscillation of an elastic stretched string. Furthermore, if the string is made up of the viscoelastic material of rate-type, the equation with the strong damping $-\Delta u_{t}$ appeared [2]. Since $\epsilon=0$, the Equation (1.1) became the following strongly damped semi-linear wave equation

$$
u_{t t}-\Delta u-\Delta u_{t}+f\left(u_{t}\right)+h(u)=g(x)
$$


which described the thermal evolution and $h(u)$ denoted a source term depending nonlinearly on displacement, $f\left(u_{t}\right)$ denoted a nonlinearly temperature-dependent internal source term [3]. With different conditions about the growth exponents $q$ and $p$ of the nonlinearities $f\left(u_{t}\right)$ and $h(u)$, some scholars [4] [5] analyzed the longtime behaviour of solutions of (1.3)-(1.2) by the global and exponential attractors in a bounded region of $\mathbb{R}^{3}$. When the nonlinearities are of fully supercritical growth, which lead to that the weak solutions of the equation lose their uniqueness. Z. J. Yang and Z. M. Liu [6] established the existence of global attractor for the subclass of limit solutions of (1.3)-(1.2) by using J. Ball's attractor theory on the generalized semiflow. Recently, I. Chueshov [7] founded that the Kirchhoff wave equation with strong nonlinear damping was still well-posed and the related evolution semigroup had a finite-dimensional global attractor in $H=H_{0}^{1} \cap L^{p+1} \times L^{2}$ in the sense of "partially strong topology". Without "partially strong topology", P. Y. Ding, Z. J. Yang [8] proved the existence of a finite-dimensional global attractor in the natural energy space. And H. L. Ma and C. K. Zhong [9] proved that global attractors for the Kirchhoff equations with strong nonlinear damping attracted $H=H_{0}^{1} \times L^{2}$ -bounded set with respect to the $H_{0}^{1} \times H_{0}^{1}$ norm.

Since $\epsilon>0$, the following quasi-linear wave equation of Kirchhoff type

$$
u_{t t}-\left(1+\|\nabla u(t)\|^{2}\right) \Delta u+u_{t}+g(u)=f(x)
$$

was studied by M. Nakao, and the author proved the existence and absorbing properties of attractors in a local sense [10]. Replacing $u_{t}$ with $-\Delta u_{t}, \mathrm{Y} . \mathrm{H}$. Wang and C. K. Zhong [11] proved the upper semicontinuity of pullback attractors in non-autonomous case. Then Z. J. Yang and Y. Q. Wang [12] studied the longtime behavior of the Kirchhoff type equation with a strong dissipation and proved that the continuous semigroup $S(t)$ possessed global attractors in the phase spaces with low regularity. As for the Kirchhoff wave equation with strong damping and critical nonlinearities, Z. J. Yang and F. Da [13] also studied the stability for the Kirchhoff wave equation with strong damping and critical nonlinearities and proved the existence of global attractors and exponential attractors. Comparing with many researches about the longtime dynamic behavior of solutions for the Kirchhoff wave equation with different types of dissipations [14]-[23], there are few researches about problem of (1.1)-(1.2). And the attractor is a key point for studying these properties, we introduce readers to see the classical book [24].

Based on these, the purpose of this paper is to prove the global attractor of problem (1.1)-(1.2), which attracts every $H_{0}^{1}(\Omega) \times L^{2}(\Omega)$-bounded set that is compacted in $H^{2}(\Omega) \times H_{0}^{1}(\Omega)$ by the way in ([25], Theorem 3.1). And we also establish the asymptotic compactness of the global attractor by operator decomposition technique ([24], Theorem 1.1). So these jobs provide a way to research the longtime dynamic behaviour of such Kirchhoff wave equations, and also reflect the strong damped properties of $\Delta u_{t}$ to some extent. 
The paper is arranged as follows. In Section 2, we verify some preliminaries. In Section 3, we prove the existence of the global attractor. In Section 4, we prove the regularity of the global attractor.

\section{Preliminaries}

Let $A=-\Delta$ on $L^{2}(\Omega)$ with $D(\underset{m}{A})=H^{2} \cap H_{0}^{1}$, and $A$ strictly positive on $H_{0}^{1}$. We define the spaces $H^{m}=D\left(A^{\frac{m}{2}},(m \in \mathbb{R})\right.$ are Hilbert spaces with the following scalar products and the norms

$$
\langle u, v\rangle_{m}=\left\langle A^{\frac{m}{2}} u, A^{\frac{m}{2}} v\right\rangle, \quad\|u\|_{H^{m}}=\left\|A^{\frac{m}{2}} u\right\| .
$$

Let $\lambda_{1}\left(>0, \lambda<\lambda_{1}\right)$ be the first eigenvalue of $A$, then $B=A-\lambda I$ with $D(B)=D(A)$.

We define the phase space $X=H_{0}^{1} \times L^{2}$ with usual graph norm. Let $\varphi(\xi)=f(\xi)+\lambda \xi$, then problem (1.1)-(1.2) becomes

$$
\begin{gathered}
u_{t t}+\left(1+\epsilon\left\|A^{\frac{1}{2}} u\right\|^{2}\right) A u+B u_{t}+\varphi\left(u_{t}\right)+h(u)=g, \\
u(0)=u_{0}, \quad u_{t}(0)=u_{1} .
\end{gathered}
$$

For any $s>r$, we have the continuous embeddings $H^{s} \hookrightarrow H^{r}$,

$$
H^{s} \hookrightarrow L^{\frac{6}{3-2 s}}(\Omega), \forall s \in\left[0, \frac{3}{2}\right),
$$

and the following inequalities hold true:

Interpolation inequality: if $r=\theta s+(1-\theta) q$, where $r, s, q \in \mathbb{R}, s \geq q$ and $\theta \in[0,1]$, then there exists a constant $C>0$ such that

$$
\|u\|_{r} \leq C\|u\|_{s}^{\theta}\|u\|_{q}^{1-\theta}, \quad \forall u \in H^{s} .
$$

The Generalized Poincare inequality:

$$
\lambda_{1}\|u\|_{\alpha}^{2} \leq\|u\|_{\alpha+1}^{2}, \quad \forall u \in H^{\alpha+1}
$$

where $\lambda_{1}>0$ is the first eigenvalue of $A$.

The Young's inequality with $\varepsilon$ : Let $a>0, b>0, \varepsilon>0, p>1, q>1$, and $\frac{1}{p}+\frac{1}{q}=1$, then

$$
a b \leq \frac{\varepsilon a^{p}}{p}+\frac{\varepsilon^{-\frac{q}{p}} b^{q}}{q},
$$

especially, when $p=q=2$, then

$$
a b \leq \varepsilon a^{2}+\frac{b^{2}}{4 \varepsilon} .
$$

The Gronwall inequality (differential form): let $\eta(\cdot)$ is nonnegative continuous differentiable function (or nonnegative absolutely continuous function), 
and satisfy

$$
\eta^{\prime}(t) \leq \phi(t) \eta(t)+\varphi(t), \quad t \in[0, T],
$$

here $\phi(t), \varphi(t)$ are nonnegative integrable functions, then

$$
\eta(t) \leq \mathrm{e}^{\int_{0}^{t} \phi(s) \mathrm{d} s}\left[\eta(0)+\int_{0}^{t} \varphi(s) \mathrm{d} s\right], \quad \forall t \in[0, T] .
$$

Throughout this paper, we will denote by $C$ a positive constant which is various in different line or even in the same line and use the following abbreviations:

$$
L^{p}=L^{p}(\Omega), \quad\|\cdot\|=\|\cdot\|_{L^{2}}, \quad\|u\|_{m}=\|u\|_{H^{m}}, \quad\|u\|_{1}=\|u\|_{H_{0}^{1}}
$$

with $p \geq 1$.

\section{Assumption 2.1.}

1) $\varphi \in C^{1}(\mathbb{R}), \varphi(0)=0$, and

$$
0 \leq \varphi^{\prime}(s) \leq C\left(1+|s|^{q-1}\right), s \in \mathbb{R},
$$

where $1 \leq q \leq p^{*} \equiv \frac{N+2}{N-2}=5$ if $N=3$

2) $h \in C^{1}(\mathbb{R}), h(0)=0$,

$$
\liminf _{|s| \rightarrow \infty} h^{\prime}(s)>-\lambda_{1},\left|h^{\prime}(s)\right| \leq C\left(1+|s|^{p-1}\right), s \in \mathbb{R},
$$

where $1 \leq p \leq p^{*}=5$ if $N=3$.

3)

$$
g \in L^{2},\left(u_{0}, u_{1}\right) \in X \text { with }\left\|\left(u_{0}, u_{1}\right)\right\|_{X} \leq R
$$

Definition 2.2. Let $S(t)_{t \geq 0}$ be a semigroup on a metric space $(E, d)$. A subset $A$ of $E$ is called a global attractor for the semigroup, if $A$ is compact and enjoys the following properties:

1) $A$ is invariant, i.e. $S(t) A=A, \forall t \geq 0$;

2) $A$ attracts all bounded set of $E$. That is, for any bounded subset $B$ of $E$,

$$
\operatorname{dist}(S(t) B, A) \rightarrow 0 \text {, as } t \rightarrow 0 .
$$

Next we only formulate the following results, which is proved in [13]:

Lemma 2.3. Let (2.11)-(2.13) be valid. Then problem (2.2)-(2.3) admits a unique weak solution $u$, with $\left(u, u_{t}\right) \in L^{\infty}\left(\mathbb{R}^{+} ; X\right) \cap C\left(\mathbb{R}^{+} ; X\right)$, $u_{t} \in L^{2}\left(\mathbb{R}^{+} ; H_{0}^{1}\right)$. Moreover, this solution possesses the following properties:

(Dissipativity)

$$
\left\|\left(u, u_{t}\right)(t)\right\|_{X}^{2}+\int_{t}^{\infty}\left(\left\|u_{t}(\tau)\right\|_{H_{0}^{1}}^{2}+\left(\varphi\left(u_{t}\right), u_{t}\right)\right) \mathrm{d} \tau \leq C(R) \mathrm{e}^{-k t}+C_{0}, \quad t \geq 0,
$$

where $k$ denotes a small positive constant, $C(R)$ and $C_{0}=C\left(\|f\|_{H^{-1}}\right)$ are positive constants.

Lemma 2.4. Let (2.11)-(2.13) be valid and when $p=5, h \in C^{2}(\mathbb{R})$. Then

$$
\left\|u_{t}(t)\right\|_{H_{0}^{1}}^{2}+\left\|u_{t t}(t)\right\|_{L^{2}}^{2} \leq R_{0}^{2}, t>0 .
$$

Actually, by exploiting (2.11) and (2.14), we can get $u, u_{t}$ are respectively 
bounded in $H_{0}^{1}, L^{2}$.

\section{Existence of Global Attractors in $H_{0}^{1} \times L^{2}$}

For every fixed $x \in B_{0}$, we split the solution $S(t) x=\left(u(t), u_{t}(t)\right)$ into the sum $\hat{\eta}(t)+\hat{\zeta}(t)$, where $\hat{\eta}(t)=\left(\hat{v}(t), \hat{v}_{t}(t)\right)$ and $\hat{\zeta}(t)=\left(\hat{w}(t), \hat{w}_{t}(t)\right)$ solve the Cauchy problems

$$
\begin{aligned}
& \left\{\begin{array}{l}
\hat{v}_{t t}+\left(1+\epsilon\left\|A^{\frac{1}{2}} \hat{u}\right\|^{2}\right) A \hat{v}+B \hat{v}_{t}+\varphi_{0}\left(\hat{v}_{t}\right)+h_{0}(\hat{v})=0, \\
\hat{\eta}(0)=x,
\end{array}\right. \\
& \left\{\begin{array}{l}
\hat{w}_{t t}+\left(1+\epsilon\left\|A^{\frac{1}{2}} \hat{u}\right\|^{2}\right) A \hat{w}+B \hat{w}_{t}=\hat{\rho} \\
\hat{\zeta}(0)=0
\end{array}\right.
\end{aligned}
$$

here

$$
\hat{\rho}=g-\left[\varphi_{0}\left(u_{t}\right)+h_{0}(u)\right]+\left[\varphi_{0}\left(\hat{v}_{t}\right)+h_{0}(\hat{v})\right]+\left[\varphi_{1}\left(u_{t}\right)+h_{1}(u)\right] .
$$

Having set $\varphi\left(u_{t}\right)+h(u)=\left[\varphi_{0}\left(u_{t}\right)+h_{0}(u)\right]+\left[\varphi_{1}\left(u_{t}\right)+h_{1}(u)\right]$, and satisfying

$$
\begin{aligned}
& \varphi_{0}\left(u_{t}\right) u_{t} \geq 0, \quad \varphi_{0}^{\prime}\left(u_{t}\right) \geq C, \quad\left|\varphi_{0}\left(u_{t}\right)-\varphi_{0}\left(v_{t}\right)\right| \leq C\left|u_{t}-v_{t}\right|\left(\left|u_{t}\right|^{4}+\left|v_{t}\right|^{4}\right), \\
& \left|\varphi_{1}\left(u_{t}\right)\right| \leq C\left(1+\left|u_{t}\right|\right) . \\
& h_{0}(u) u \geq 0, \quad\left|h_{0}(u)-h_{0}(v)\right| \leq C|u-v|\left(|u|^{4}+|v|^{4}\right), \quad\left|h_{1}(u)\right| \leq C(1+|u|) .
\end{aligned}
$$

From now on, $c_{0}, v_{0}>0$ and $J_{0}$ will denote generic constants and a generic function, respectively, depending only on $B_{0}$.

Theorem 3.1. Let (2.11)-(2.13) be valid, then the solution semigroup $S(t)$ possesses a global attractor $\mathcal{B}$ in $X$.

Proof. Estimate (2.14) shows

$$
\left\|\left(u, u_{t}\right)(t)\right\|_{X}^{2} \leq C(R) \mathrm{e}^{-k t}+C_{0}, t \geq 0,
$$

such that the ball $B_{0}=\left\{\left(u, u_{t}\right) \in X \mid\left\|\left(u, u_{t}\right)\right\|_{X} \leq R_{0}\right\}$ is an absorbing set of the semigroup $S(t)$ in $X$ for $R_{0}>C_{0}\left(\|g\|_{H^{-1}}\right)$.

In order to prove the existence of the global attractors, now we need to prove the asymptotic compactness.

Multiplying the first equation of (3.1) by $\hat{v}_{t}+\gamma \hat{v}$ and integrating over $\Omega$, we get

$$
\left\langle\hat{v}_{t t}+\left(1+\epsilon\left\|A^{\frac{1}{2}} \hat{u}\right\|^{2}\right) A \hat{v}+B \hat{v}_{t}+\varphi_{0}\left(\hat{v}_{t}\right)+h_{0}(\hat{v}), \hat{v}_{t}+\gamma \hat{v}\right\rangle=0 .
$$

By using $\epsilon \geq 0, \varphi_{0}\left(u_{t}\right) u_{t} \geq 0, h_{0}(u) u \geq 0$ and the generalized Poincare inequality, then

$$
\begin{aligned}
& \frac{1}{2} \frac{\mathrm{d}}{\mathrm{d} t}\left[\left\|\hat{v}_{t}\right\|^{2}+\|\hat{v}\|_{1}^{2}+\gamma\left(\|\hat{v}\|_{1}^{2}+\left\langle\hat{v}_{t}, \hat{v}\right\rangle-\lambda\|\hat{v}\|^{2}\right)\right] \\
& \leq\left(\gamma-\lambda_{1}+\lambda\right)\left\|\hat{v}_{t}\right\|^{2}-\gamma\|\hat{v}\|_{1}^{2}-\gamma \int_{\Omega} \varphi_{0}\left(\hat{v}_{t}\right) \hat{v} \mathrm{~d} x-\int_{\Omega} h_{0}(\hat{v}) \hat{v}_{t} \mathrm{~d} x,
\end{aligned}
$$

By $\lambda<\lambda_{1}$, we know 


$$
\begin{aligned}
& \frac{1}{2} \frac{\mathrm{d}}{\mathrm{d} t}\left[\left\|\hat{v}_{t}\right\|^{2}+\|\hat{v}\|_{1}^{2}+\gamma\left(\|\hat{v}\|_{1}^{2}+\left\langle\hat{v}_{t}, \hat{v}\right\rangle-\lambda\|\hat{v}\|^{2}\right)\right] \\
& \geq \frac{1}{2} \frac{\mathrm{d}}{\mathrm{d} t}\left[\left\|\hat{v}_{t}\right\|^{2}+\|\hat{v}\|_{1}^{2}+\gamma\left(\left\langle\hat{v}_{t}, \hat{v}\right\rangle-\lambda_{1}\|\hat{v}\|^{2}\right)\right],
\end{aligned}
$$

where $\gamma>0$ is small enough such that

$$
E(t)=\frac{1}{2}\left[\left\|\hat{v}_{t}\right\|^{2}+\|\hat{v}\|_{1}^{2}+\gamma\left(\left\langle\hat{v}_{t}, \hat{v}\right\rangle-\lambda_{1}\|\hat{v}\|^{2}\right)\right] \sim \frac{1}{2}\left[\left\|\hat{v}_{t}\right\|^{2}+\|\hat{v}\|_{1}^{2}\right] .
$$

Actually, noting that $\varphi_{0}^{\prime}\left(\hat{v}_{t}\right) \geq C$, and by exploiting (2.8) and (2.12), we deduce that

$$
\begin{aligned}
-\int_{\Omega} \varphi_{0}\left(\hat{v}_{t}\right) \hat{v} \mathrm{~d} x & =-\int_{\Omega} \frac{\varphi_{0}\left(\hat{v}_{t}\right)-\varphi(0)}{\hat{v}_{t}-0} \hat{v}_{t} \hat{v} \mathrm{~d} x \\
& =-\int_{\Omega} \varphi_{0}^{\prime}\left(\hat{v}_{t}\right) \hat{v}_{t} \hat{v} \mathrm{~d} x \\
& \leq-C\left\langle\hat{v}_{t}, \hat{v}\right\rangle,
\end{aligned}
$$

and

$$
-\int_{\Omega} h_{0}(\hat{v}) \hat{v}_{t} \mathrm{~d} x \leq \int_{\Omega} \lambda_{1} \hat{v}_{t} \hat{v} \mathrm{~d} x \leq \frac{1}{2 \gamma}\|\hat{v}\|^{2}+\frac{\gamma \lambda_{1}}{2}\left\|\hat{v}_{t}\right\|^{2} .
$$

From (3.5)-(3.7), we get

$$
\frac{\mathrm{d}}{\mathrm{d} t} E(t) \leq\left(\gamma+\lambda-\lambda_{1}+\frac{\gamma \lambda_{1}}{2}\right)\left\|\hat{v}_{t}\right\|^{2}-\gamma\|\hat{v}\|_{1}^{2}+\frac{1}{2 \gamma}\|\hat{v}\|^{2}-C\left\langle v_{t}, v\right\rangle \leq-C E(t),
$$

where $\gamma>0$ is small enough such that $\left(\gamma+\lambda-\lambda_{1}+\frac{\gamma \lambda_{1}}{2}\right)$ is negative. Furthermore, by the Gronwall inequality, we can get

$$
\|\hat{\eta}(t)\|_{H_{0}^{1} \times L^{2}} \leq c_{0} \mathrm{e}^{-v_{0} t}\|x\|_{H_{0}^{1} \times L^{2}} .
$$

Next multiplying the first equation of (3.2) by $A^{\frac{1}{4}} \hat{\omega}_{t}+\gamma A^{\frac{1}{4}} \hat{\omega}$ and integrating over $\Omega$, we get

$$
\begin{aligned}
& \|\left\langle\hat{w}_{t t}+\left(1+\epsilon\left\|A^{\frac{1}{2}} u\right\|^{2}\right) A \hat{w}+B \hat{w}_{t}, A^{\frac{1}{4}} \hat{w}_{t}+\gamma A^{\frac{1}{4}} \hat{w}\right\rangle \\
& =\frac{\mathrm{d}}{\mathrm{d} t}\left[\frac{1}{2}\left\|\hat{w}_{t}\right\|_{\frac{1}{4}}^{2}+\frac{1}{2}\|\hat{w}\|_{\frac{5}{4}}^{2}+\gamma\left(\frac{1}{2}\|\hat{w}\|_{\frac{5}{4}}^{2}-\frac{\lambda}{2}\|\hat{w}\|_{\frac{1}{4}}^{2}+\left\langle\hat{w}_{t}, A^{\frac{1}{4}} \hat{w}\right\rangle\right)\right] \\
& +\epsilon\left\|A^{\frac{1}{2}} u\right\|^{2}\left\langle A \hat{w}, A^{\frac{1}{4}} \hat{w}_{t}\right\rangle+\left\|\hat{w}_{t}\right\|_{\frac{5}{4}}^{2}-\lambda\left\|\hat{w}_{t}\right\|_{\frac{1}{4}}^{2}-\gamma\left\|\hat{w}_{t}\right\|_{\frac{1}{4}}^{2}+\gamma\|\hat{w}\|_{\frac{5}{4}}^{2} \\
& +\epsilon \gamma\left\|A^{\frac{1}{2}} u\right\|^{2}\left\langle A \hat{w}, A^{\frac{1}{4}} \hat{w}\right\rangle \\
& \geq \frac{\mathrm{d}}{\mathrm{d} t}\left(\frac{1}{2}\left\|\hat{w}_{t}\right\|_{\frac{1}{4}}^{2}+\frac{1}{2}\|\hat{w}\|_{\frac{5}{4}}^{2}\right)+\left\|\hat{w}_{t}\right\|_{\frac{5}{4}}^{2}-\lambda\left\|\hat{w}_{t}\right\|_{\frac{1}{4}}^{2}-\gamma\left\|\hat{w}_{t}\right\|_{\frac{1}{4}}^{2}+\gamma\|\hat{w}\|_{\frac{5}{4}}^{2},
\end{aligned}
$$

where $\gamma>0$ is small enough. Then we define the energy functional 


$$
E_{1}(t)=\frac{1}{2}\left(\left\|\hat{w}_{t}\right\|_{\frac{1}{4}}^{2}+\|\hat{w}\|_{\frac{5}{4}}^{2}\right)
$$

At the same time, by the interpolation inequality, we have

$$
\begin{aligned}
\|\hat{\rho}\|_{L^{3}(\Omega)}^{\frac{4}{3}}= & \left\|g+\left(\varphi_{0}\left(\hat{v}_{t}\right)-\varphi_{0}\left(u_{t}\right)\right)+\left(h_{0}(\hat{v})-h_{0}(u)\right)+\left(\varphi_{1}\left(u_{t}\right)+h_{1}(u)\right)\right\| \\
\leq & C\|g\|+C\|\hat{w}\|_{\frac{5}{4}}\left(\|\hat{v}\|_{1}^{4}+\|u\|_{1}^{4}\right)+C\left\|\hat{w}_{t}\right\|_{\frac{5}{4}}\left(\left\|\hat{v}_{t}\right\|_{1}^{4}+\left\|u_{t}\right\|_{1}^{4}\right) \\
& +C\left(1+\left\|u_{t}\right\|_{1}\right)+C\left(1+\|u\|_{1}\right) \\
\leq & c_{0} \mathrm{e}^{-v_{0} t}\|x\|_{H^{1} \times L^{2}}^{2}\|\hat{w}\|_{\frac{5}{4}}+c \mathrm{e}^{-k t}\left\|\hat{w}_{t}\right\|_{\frac{5}{4}}+C\left(\|g\|+\|u\|_{1}+\left\|u_{t}\right\|_{1}+1\right) \\
\leq & c_{0} \mathrm{e}^{-v_{0} t}\|\hat{w}\|_{\frac{5}{4}}+c \mathrm{e}^{-k t}\left\|\hat{w}_{t}\right\|_{\frac{5}{4}}+C\left(1+\mathrm{e}^{-k t}\right),
\end{aligned}
$$

and by the embedding $H^{\frac{3}{4}} \hookrightarrow L^{4}$, then

$$
\begin{aligned}
& \|\left\langle\hat{\rho}, A^{\frac{1}{4}} \hat{w}_{t}+\gamma A^{\frac{1}{4}} \hat{w}\right\rangle \mid \\
& \leq\|\hat{\rho}\|_{L^{\frac{4}{3}}}\left(\left\|A^{\frac{1}{4}} \hat{w}_{t}\right\|_{L^{4}}+\gamma\left\|A^{\frac{1}{4}} \hat{w}\right\|_{L^{4}}\right) \\
& \leq\|\hat{\rho}\|\left(\left\|A^{\frac{1}{4}} \hat{w}_{t}\right\|_{\frac{3}{4}}+\gamma\left\|A^{\frac{1}{4}} \hat{w}\right\|_{\frac{3}{4}}\right) \\
& \leq c_{0} \mathrm{e}^{-v_{0} t}\|\hat{w}\|_{\frac{5}{4}}^{2}+c \mathrm{e}^{-k t}\left\|\hat{w}_{t}\right\|_{\frac{5}{4}}^{2}+\delta\left\|\hat{w}_{t}\right\|_{\frac{5}{4}}^{2}+\gamma \delta\|\hat{w}\|_{\frac{5}{4}}^{2}+C\left(1+\mathrm{e}^{-k t}\right) .
\end{aligned}
$$

By exploiting (2.8) and the generalized Poincare inequality, from (3.9)-(3.11), we get

$$
\begin{aligned}
\frac{\mathrm{d}}{\mathrm{d} t} E_{1}(t) \leq & \left(c_{0} \mathrm{e}^{-k t}+\delta-1\right)\left\|\hat{w}_{t}\right\|_{\frac{5}{4}}^{2}+(\lambda+\gamma)\left\|\hat{w}_{t}\right\|_{\frac{1}{4}}^{2} \\
& +\left(c_{0} \mathrm{e}^{-v_{0} t}+\gamma \delta-\gamma\right)\|\hat{w}\|_{\frac{5}{4}}^{2}+C\left(1+\mathrm{e}^{-k t}\right) \\
\leq & -C \lambda_{1}\left\|\hat{w}_{t}\right\|_{\frac{1}{4}}^{2}+(\lambda+\gamma)\left\|\hat{w}_{t}\right\|_{\frac{1}{4}}^{2}+\left(c_{0} \mathrm{e}^{-v_{0} t}-C\right)\|\hat{w}\|_{\frac{5}{4}}^{2}+C\left(1+\mathrm{e}^{-k t}\right) \\
\leq & -\left(C-c_{0} \mathrm{e}^{-v_{0} t}\right) E_{1}(t)+C\left(1+\mathrm{e}^{-k t}\right),
\end{aligned}
$$

where $\delta>0$ is small enough and by $\lambda<\lambda_{1}$, we get $\left(-C \lambda_{1}+\lambda+\gamma\right),(\gamma \delta-\gamma)$ are negative. Then from the Gronwall inequality and noting that $\hat{\zeta}(0)=\left(\hat{\omega}(0), \hat{\omega}_{t}(0)\right)=0$, we get

$$
E_{1}(t) \leq c_{0} e^{-v t} E_{1}(0)+C\left(1+\mathrm{e}^{-k t}\right) \leq C\left(1+\mathrm{e}^{-k t}\right)
$$

which provides the following estimate

$$
\|\hat{\zeta}(t)\|_{H^{\frac{5}{4}} \times H^{\frac{1}{4}}} \leq C\left(1+\mathrm{e}^{-k t}\right),
$$

From (3.8) and (3.12), we obtain that the evolution semigroup $S(t)$ is asymptotically compact in $X$, so the solution semigroup $S(t)$ possesses a global attractor $\mathcal{B}$ in $H_{0}^{1} \times L^{2}$, which 


$$
\mathcal{B}=\bigcap_{t_{0} \geq 0 t \geq t_{0}} S(t) B_{0},
$$

where $t_{0}>0$ is chosen such that $S(t) B_{0} \subset B_{0}$ for $t \geq t_{0}$.

\section{Regularity of Global Attractors}

Now we are in a position to state and prove the main result:

Theorem 4.1. The attractor $\mathcal{B}$ of the semigroup $S(t)$ on $X$ is bounded in $H^{2} \times H_{0}^{1}$.

Proof. Having set $x=y+z$. For $y \in B_{0}, z \in H^{2} \times H_{0}^{1}$, we split the solution into the sum

$$
S(t) x=Y(t) y+Z(t) z
$$

where $\eta(t)=Y(t) y=\left(v(t), v_{t}(t)\right)$ and $\zeta(t)=Z(t) z=\left(w(t), w_{t}(t)\right)$ solve the following equations with initial data $\eta(0)=y, \zeta(0)=z$,

$$
\left\{\begin{array}{l}
v_{t t}+\left(1+\epsilon\left\|A^{\frac{1}{2}} u\right\|^{2}\right) A v+B v_{t}=0, \\
\eta(0)=x,
\end{array}\right.
$$

and

$$
\left\{\begin{array}{l}
w_{t t}+\left(1+\epsilon\left\|A^{\frac{1}{2}} u\right\|^{2}\right) A w+B w_{t}=\rho, \\
\zeta(0)=0,
\end{array}\right.
$$

where $\rho(t)=g+\varphi\left(u_{t}\right)+h(u)$.

Multiplying the first equation of (4.1) by $v_{t}+\gamma \nu$ and integrating over $\Omega$, by $\epsilon>0$ we get

$$
\frac{1}{2} \frac{\mathrm{d}}{\mathrm{d} t}\left[\left\|v_{t}\right\|^{2}+\|v\|_{1}^{2}+\frac{\gamma}{2}\left(\|v\|_{1}^{2}-\lambda\|v\|^{2}+\left\langle v_{t}, v\right\rangle\right)\right]+\gamma\|v\|_{1}^{2}+\left\|v_{t}\right\|_{1}^{2}-\lambda\left\|v_{t}\right\|^{2} \leq 0,
$$

where $\gamma>0$ is small enough such that

$$
E_{2}(t)=\frac{1}{2}\left[\left\|v_{t}\right\|^{2}+\|v\|_{1}^{2}+\frac{\gamma}{2}\left(\|v\|_{1}^{2}-\lambda\|v\|^{2}+\left\langle v_{t}, v\right\rangle\right)\right] \sim \frac{1}{2}\left(\left\|v_{t}\right\|^{2}+\|v\|_{1}^{2}\right) .
$$

By $\lambda<\lambda_{1}$ and the generalized Poincare inequality, we deduce that

$$
\frac{\mathrm{d}}{\mathrm{d} t} E_{2}(t) \leq\left(\lambda-\lambda_{1}\right)\left\|v_{t}\right\|^{2}-\gamma\|v\|_{1}^{2} \leq-\frac{C}{2}\left(\left\|v_{t}\right\|^{2}+\|v\|_{1}^{2}\right),
$$

then by the Gronwall inequality, we get

$$
\|\eta(t)\|_{H_{0}^{1} \times L^{2}} \leq c_{0} \mathrm{e}^{-v_{0} t}\|y\|_{H_{0}^{1} \times L^{2}} .
$$

Next multiplying the first equation of (4.2) by $A w_{t}+\gamma A w$ and integrating over $\Omega$, exploiting (2.8) and the Hölder's inequality, the right side becomes 


$$
\begin{aligned}
& \left\langle\left\langle\rho, A w_{t}+\gamma A w\right\rangle\right| \\
\leq & \left|\left\langle g, A w_{t}+\gamma A w\right\rangle\right|+\left|\left\langle\varphi\left(u_{t}\right), A w_{t}+\gamma A w\right\rangle\right|+\left|\left\langle h(u), A w_{t}+\gamma A w\right\rangle\right| \\
\leq & \frac{1}{2 \delta}\|g\|^{2}+\delta\left\|w_{t}\right\|_{2}^{2}+\int_{\Omega} C\left(\left|u_{t}\right|+\left|u_{t}\right|^{5}\right)\left|A w_{t}\right| \mathrm{d} x+\int_{\Omega} C\left(|u|+|u|^{5}\right)\left|A w_{t}\right| \mathrm{d} x \\
& +\gamma\left[\delta\|w\|_{2}^{2}+\int_{\Omega} C\left(\left|u_{t}\right|+\left|u_{t}\right|^{5}\right)|A w| \mathrm{d} x+\int_{\Omega} C\left(|u|+|u|^{5}\right)|A w| \mathrm{d} x\right] \\
\leq & \frac{1}{2 \delta}\|g\|^{2}+\delta\left\|w_{t}\right\|_{2}^{2}+\int_{\Omega} C\left(1+\left|u_{t}\right|^{6}\right)\left|A w_{t}\right| \mathrm{d} x+\int_{\Omega} C\left(1+|u|^{6}\right)\left|A w_{t}\right| \mathrm{d} x \\
& +\gamma\left[\delta\|w\|_{2}^{2}+\int_{\Omega} C\left(1+\left|u_{t}\right|^{6}\right)|A w| \mathrm{d} x+\int_{\Omega} C\left(1+|u|^{6}\right)|A w| \mathrm{d} x\right] \\
\leq & \frac{1}{2 \delta}\|g\|^{2}+C \delta \gamma\|w\|_{2}^{2}+C \delta\left\|w_{t}\right\|_{2}^{2}+\frac{C}{2 \delta}\left(\|u\|_{L^{2}}^{12}+\left\|u_{t}\right\|_{L^{2}}^{12}+|\Omega|\right) \\
\leq & C \delta \gamma\|w\|_{2}^{2}+C \delta\left\|w_{t}\right\|_{2}^{2}+J_{0}(t),
\end{aligned}
$$

where $\delta>0$ is small enough, we know $J_{0}(t)$ is bounded by (2.13) and lemma 2.3. At the same time, the left side becomes

$$
\begin{aligned}
& \|\left\langle w_{t t}+\left(1+\epsilon\left\|A^{\frac{1}{2}} u\right\|^{2}\right) A w+B w_{t}, A w_{t}+\gamma A w\right\rangle \\
& =\frac{\mathrm{d}}{\mathrm{d} t}\left[\frac{1}{2}\left\|w_{t}\right\|_{1}^{2}+\frac{1}{2}\|w\|_{2}^{2}+\gamma\left(\frac{1}{2}\|w\|_{2}^{2}-\frac{\lambda}{2}\|w\|_{1}^{2}+\left\langle w_{t}, A w\right\rangle\right)\right] \\
& +\epsilon\left\|A^{\frac{1}{2}} u\right\|^{2}\left\langle A w, A w_{t}\right\rangle+\left\|w_{t}\right\|_{2}^{2}-\lambda\left\|w_{t}\right\|_{1}^{2} \\
& +\epsilon \gamma\left\|A^{\frac{1}{2}} u\right\|^{2}\left\langle A w, A w_{t}\right\rangle+\gamma\|w\|_{2}^{2}-\gamma\left\|w_{t}\right\|_{1}^{2} \\
& \geq \frac{\mathrm{d}}{\mathrm{d} t}\left(\frac{1}{2}\left\|w_{t}\right\|_{1}^{2}+\frac{1}{2}\|w\|_{2}^{2}\right)+\left\|w_{t}\right\|_{2}^{2}-\lambda\left\|w_{t}\right\|_{1}^{2}+\gamma\|w\|_{2}^{2}-\gamma\left\|w_{t}\right\|_{1}^{2},
\end{aligned}
$$

then we define the energy functional

$$
E_{3}(t)=\frac{1}{2}\left[\left\|w_{t}\right\|_{1}^{2}+\|w\|_{2}^{2}+\gamma\left(\|w\|_{2}^{2}-\lambda\|w\|_{1}^{2}+\left\langle w_{t}, A w\right\rangle\right)\right],
$$

where $\gamma>0$ is small enough such that $E_{3}(t) \sim \frac{1}{2}\left(\left\|w_{t}\right\|_{1}^{2}+\|w\|_{2}^{2}\right)$. By combining (4.5)-(4.7) and the embedding $H^{2} \hookrightarrow H_{0}^{1}$, we get

$$
\begin{aligned}
\frac{\mathrm{d}}{\mathrm{d} t} E_{3}(t) & \leq(C \delta-1)\left\|w_{t}\right\|_{2}^{2}+(C \gamma \delta-\gamma)\|w\|_{2}^{2}+(\lambda+\gamma)\left\|w_{t}\right\|_{1}^{2}+J_{0}(t) \\
& \leq\left(-C \lambda_{1}+\lambda+\gamma\right)\left\|w_{t}\right\|_{1}^{2}-C\|w\|_{2}^{2}+J_{0}(t) \\
& \leq-\frac{C}{2}\left(\left\|w_{t}\right\|_{1}^{2}+\|w\|_{2}^{2}\right)+J_{0}(t),
\end{aligned}
$$

where $\delta>0$ is small enough and by $\lambda<\lambda_{1}$, we get $\left(-C \lambda_{1}+\lambda+\gamma\right),(C \gamma \delta-\gamma)$ are negative. From the Gronwall inequality, we get

$$
E_{3}(t) \leq c_{0} \mathrm{e}^{-v t} E_{3}(0)+J_{0}(t),
$$

which provides the estimate 


$$
\|\zeta(t)\|_{H^{2} \times H_{0}^{1}} \leq c_{0} \mathrm{e}^{-v t}\|z\|_{H^{2} \times H_{0}^{1}}+J_{0}(t) .
$$

From (4.4) and (4.8), for every bounded set $B \subset H_{0}^{1} \times L^{2}$, we get

$$
\operatorname{dist}_{H_{0}^{1} \times L^{2}}\left(S(t) B, B_{H^{2} \times H_{0}^{1}}\left(J_{0}(t)\right)\right) \leq C \mathrm{e}^{-v_{0} t} \rightarrow 0 \text {, as } t \rightarrow \infty,
$$

so

$$
S(t) B \subseteq B_{H^{2} \times H_{0}^{1}}\left(J_{0}(t)\right), \forall t \leq t_{0} .
$$

Then we finish the proof.

\section{Conclusion}

In this paper, we first prove that the Kirchhoff wave equation with strong damping and critical nonlinearities possesses a global attractor in $H_{0}^{1}(\Omega) \times L^{2}(\Omega)$. Then we split the solution into two parts, one part decays exponentially and the other part satisfies asymptotic behaviour in spaces with higher regularity. By the operator decomposition technique, we get the global attractor which is compactly bounded in $H^{2}(\Omega) \times H_{0}^{1}(\Omega)$.

\section{Conflicts of Interest}

The authors declare no conflicts of interest regarding the publication of this paper.

\section{References}

[1] Kirchhoff, G. (1883) Vorlesungen ber Mechanik. Teubner, Stuttgart.

[2] Duvaut, G. and Lions, J.L. (1976) Inequalities in Mechanics and Physics. Springer-Verlag, Berlin, New York. https://doi.org/10.1007/978-3-642-66165-5

[3] DellOro, F. and Pata, V. (2011) Long-Term Analysis of Strongly Damped Nonlinear Wave Equations. Nonlinearity, 24, 3413-3435. https://doi.org/10.1088/0951-7715/24/12/006

[4] DellOro, F. (2013) Global Attractors for Strongly Damped Wave Equations with Subcritical-Critical Nonlinearities. Communications on Pure \& Applied Analysis, 12, 1015-1027. https://doi.org/10.3934/cpaa.2013.12.1015

[5] DellOro, F. and Pata, V. (2012) Strongly Damped Wave Equations with Critical Nonlinearities. Nonlinear Analysis, 75, 5723-5735.

https://doi.org/10.1016/j.na.2012.05.019

[6] Yang, Z.J. and Liu, Z.M. (2017) Global Attractor for a Strongly Damped Wave Equation with Fully Supercritical Nonlinearities. Discrete \& Continuous Dynamical Systems, 37, 2181-2205. https://doi.org/10.3934/dcds.2017094

[7] Chueshov, I. (2012) Long-Time Dynamics of Kirchhoff Wave Models with Strong Nonlinear Damping. Journal of Differential Equations, 252, 1229-1262. https://doi.org/10.1016/j.jde.2011.08.022

[8] Ding, P.Y., Yang, Z.J. and Li, Y. (2018) Global Attractor of the Kirchhoff Wave Models with Strong Nonlinear Damping. Applied Mathematics Letters, 76, 40-45. https://doi.org/10.1016/j.aml.2017.07.008

[9] Ma, H.L. and Zhong, C.K. (2017) Attractors for the Kirchhoff Equations with Strong Nonlinear Damping. Applied Mathematics Letters, 74, 127-133. 
https://doi.org/10.1016/j.aml.2017.06.002

[10] Nakao, M. (2009) An Attractor for a Nonlinear Dissipative Wave Equation of Kirchhoff Type. Journal of Mathematical Analysis and Applications, 353, 652-659. https://doi.org/10.1016/j.jmaa.2008.09.010

[11] Wang, Y.H. and Zhong, C.K. (2013) Upper Semicontinuity of Pullback Attractors for Nonautonomous Kirchhoff Wave Models. Discrete \& Continuous Dynamical Systems, 33, 3189-3209. https://doi.org/10.3934/dcds.2013.33.3189

[12] Yang, Z.J. and Wang, Y.Q. (2010) Global Attractor for the Kirchhoff Type Equation with a Strong Dissipation. Journal of Differential Equations, 249, 3258-3278. https://doi.org/10.1016/j.jde.2010.09.007

[13] Yang, Z.J. and Da, F. (2019) Stability of Attractors for the Kirchhoff Wave Equation with Strong Damping and Critical Nonlinearities. Journal of Mathematical Analysis and Applications, 469, 298-320. https://doi.org/10.1016/j.jmaa.2018.09.012

[14] Bae, J.J. and Nakao, M. (2004) Existence Problem of Global Solutions of the Kirchhoff Type Wave Equations with a Localized Weakly Nonlinear Dissipation in Exterior Domains. Discrete \& Continuous Dynamical Systems, 11, 731-743. https://doi.org/10.3934/dcds.2004.11.731

[15] Nishihara, K. (1993) Decay Properties of Solutions of Some Quasilinear Hyperbolic Equations with Strong Damping. Nonlinear Analysis, 21, 17-21. https://doi.org/10.1016/0362-546X(93)90174-Q

[16] Ono, K. (2011) On Decay Properties of Solutions for Degenerate Strongly Damped Wave Equations of Kirchhoff Type. Journal of Mathematical Analysis and Applications, 381, 229-239. https://doi.org/10.1016/j.jmaa.2011.03.034

[17] Ono, K. (1997) Global Existence, Decay, and Blowup of Solutions for Some Mildly Degenerate Nonlinear Kirchhoff Strings. Journal of Differential Equations, 137, 273-301. https://doi.org/10.1006/jdeq.1997.3263

[18] Cavalcanti, M.M., Cavalcanti, V.N.D., Filho, J.S.P. and Soriano, J.A. (1998) Existence and Exponential Decay for a Kirchhoff-Carrier Model with Viscosity. Journal of Mathematical Analysis and Applications, 226, 40-60. https://doi.org/10.1006/jmaa.1998.6057

[19] Ghisi, M. (2006) Global Solutions for Dissipative Kirchhoff Strings with Non-Lipschitz Nonlinear Term. Journal of Differential Equations, 230, 128-139. https://doi.org/10.1016/j.jde.2006.07.020

[20] Ghisi, M. (2016) Kirchhoff Equations with Strong Damping. Journal of Evolution Equations, 16, 441-482. https://doi.org/10.1007/s00028-015-0308-0

[21] Ancona, P.D. and Spagnolo, S. (1994) Nonlinear Perturbations of the Kirchhoff Equation. Communications on Pure and Applied Mathematics, 47, 1005-1024. https://doi.org/10.1002/cpa.3160470705

[22] Matsuyama, T. and Ikehata, R. (1996) On Global Solution and Energy Decay for the Wave Equation of Kirchhoff Type with Nonlinear Damping Term. Journal of Mathematical Analysis and Applications, 204, 729-753. https://doi.org/10.1006/jmaa.1996.0464

[23] Mizumachi, T. (1998) Time Decay of Solutions for Degenerate Kirchhoff Equation. Nonlinear Analysis, 33, 235-252. https://doi.org/10.1016/S0362-546X(97)00540-3

[24] Temam, R. (1997) Infinite-Dimensional Dynamical Systems in Mechanics and Physics. Springer, New York. https://doi.org/10.1007/978-1-4612-0645-3

[25] Conti, M. and Pata, V. (2009) On the Regularity of Global Attractors. Discrete \& Continuous Dynamical Systems, 25, 1209-1217.

https://doi.org/10.3934/dcds.2009.25.1209 